\title{
Leukocyte adhesion-GPCR EMR2 is aberrantly expressed in human breast carcinomas and is associated with patient survival
}

\author{
JOHN Q. DAVIES ${ }^{1,7 *}$, HSI-HSIEN LIN ${ }^{2 *}$, MARTIN STACEY $^{3 *}$, SIMON YONA $^{1}$, \\ GIN-WEN CHANG ${ }^{2}$, SIAMON GORDON ${ }^{1}$, JÖRG HAMANN ${ }^{4}$, LETICIA CAMPO ${ }^{5}$, \\ CHENG HAN $^{5}$, PETER CHAN ${ }^{6}$ and STEPHEN B. FOX ${ }^{5,6}$
}

\begin{abstract}
${ }^{1}$ Sir William Dunn School of Pathology, University of Oxford, Oxford, UK; ${ }^{2}$ Department of Microbiology and Immunology, Chang Gung University, Taiwan, R.O.C.; ${ }^{3}$ Institute of Molecular and Cellular Biology, University of Leeds, Leeds, UK; ${ }^{4}$ Department of Experimental Immunology, Academic Medical Center, University of Amsterdam, Amsterdam, The Netherlands; ${ }^{5}$ The Nuffield Department of Clinical Laboratory Sciences and Molecular Oncology, John Radcliffe Hospital, University of Oxford, Oxford, UK;

${ }^{6}$ Department of Pathology, Peter Macullum Cancer Centre, Melbourne, Australia
\end{abstract}

Received October 5, 2010; Accepted November 24, 2010

DOI: $10.3892 /$ or.2010.1117

\begin{abstract}
EGF-like module containing mucin-like hormone receptor 2 (EMR2) is a leukocyte-restricted adhesion $G$ protein-coupled receptor. Aberrant expression of EMR2 and its highly homologous molecule CD97 have been reported in various human cancers. Herein, we investigate the expression of EMR2 in neoplastic breast human tissue and its relationship with patient survival. EMR2 expression in normal and neoplastic breast tissue was assessed by immunohistochemistry in sections from 10 normal controls and microarrayed tissue cores from 69 cases of ductal carcinoma in situ (DCIS) and 272 invasive carcinomas. The pattern and intensity of staining was correlated with the clinicopathological characteristics of each case and the disease outcome. While absent in normal breast epithelium, EMR2 was significantly up-regulated in the cytoplasmic and nuclear compartments of both DCIS and invasive carcinoma, with invasive samples displaying significantly higher expression levels compared with in situ disease. In invasive disease, EMR2 cytoplasmic expression was significantly associated with higher tumour
\end{abstract}

Correspondence to: Dr Hsi-Hsien Lin, Department of Microbiology and Immunology, Chang Gung University, 259 Wen-Hwa 1st Road, Kwei-Shan, Taoyuan, Taiwan, R.O.C.

E-mail: hhlin@mail.cgu.edu.tw

Present address: ${ }^{7}$ Department of Anatomical Pathology, University of Cape Town, Cape Town, South Africa

*Contributed equally

Key words: adhesion-GPCR, EMR2, breast cancer, nucleus, survival grade but not with patient age, nodal status, tumour size, estrogen receptor expression, relapse-free or overall survival. In contrast, EMR2 nuclear expression correlated negatively with higher tumour grade. Of note, EMR2 nuclear expression was associated with longer relapse-free survival as well as overall survival. This study indicates that EMR2 is expressed in neoplastic breast epithelium and suggests that expression patterns of EMR2 are relevant in breast cancer progression. The association of improved patient survival with higher nuclear expression levels identifies EMR2 as a potential biomarker in patients with invasive breast cancer.

\section{Introduction}

Adhesion class G protein-coupled receptors (GPCRs) are transmembrane molecules characterized by an unusually large $\mathrm{N}$-terminal extracellular region. This extracellular region is linked to a class B GPCR-related seven transmembrane (TM7) moiety via a mucin-like stalk, resulting in a naturally occurring chimeric molecule (1). The extracellular domains (ECD) of these adhesion-GPCRs usually contain a diverse array of protein modules, such as lectin-like, immunoglobulinlike and epidermal growth factor (EGF)-like motifs, which are known to mediate protein-protein interactions (1). Current data suggest that these domains are involved in cellular adhesion, which causes/potentiates signaling via the TM7 region, resulting in cellular activation or migration (1). The EGF-TM7 receptors belong to a subfamily of adhesion-GPCRs expressed predominantly on leukocytes and are known to be responsible for a number of immune functions including host defense (2), immune tolerance (3), neutrophil migration and activation (4) and modulation of T cell activation (5).

Aside from these immune roles, the EGF-TM7 family members have been implicated in a number of non-immune functions such as angiogenesis and carcinogenesis (6). The expression of CD97, a predominantly leukocyte-restricted 
EGF-TM7 protein (7), has been reported on cancer cells in a number of human malignancies such as thyroid, colonic, pancreatic, gastric and oesophageal carcinoma (8-10), where it is thought to play a role in growth, migration and metastasis of tumour cells (11).

Furthermore, CD97 overexpression in colonic adenocarcinoma is not only associated with enhanced migration and invasive capacity of malignant cells, but also with advanced clinical stage (12), suggesting that expression of CD97, at least in colonic adenocarcinoma, has a direct functional effect during disease progression. CD97 has marked sequence homology to another EGF-TM7 receptor, EGF-like module containing mucin-like hormone receptor 2 (EMR2) (13). Although EMR2 is considered more leukocyte-restricted than CD97, it has been identified at low levels in colonic adenocarcinoma, but not in gastric, pancreatic or oesophageal carcinoma (9).

Additionally, specific isoforms of EMR2 and CD97 share a common extracellular ligand, namely chondroitin sulphate glycosaminoglycan, which is thought to mediate cell-cell and cell-matrix attachment (14) and may have some relevance in carcinomas rich in cell-surface or extracellular chondroitin sulphate. During the characterization of the EMR2-chondroitin sulphate interaction (15), we noticed that the breast carcinoma cell line SK-BR3 not only expressed EMR2ligand, but also EMR2 protein. In view of this finding, we chose to further investigate the expression of EMR2 in human breast cancer. We show that EMR2 is expressed by a number of breast cancer cell lines, is not or weakly expressed by normal breast epithelium, and is up-regulated in ductal carcinoma in situ (DCIS) and invasive breast carcinoma. We correlate immuno-histochemical staining patterns with the clinicopathological information and outcome.

\section{Materials and methods}

Breast cancer cell culture. The breast cancer lines MCF-7, T7D4, MB468, SK-BR3 and MB231 were obtained from the Nuffield Department of Laboratory Medicine, Oxford University. All cell lines were grown in DMEM (Gibco) containing $10 \%$ FCS supplemented with $2 \mathrm{mM}$ L-glutamine, in a humidified incubator with $5 \% \mathrm{CO}_{2}$ at $37^{\circ} \mathrm{C}$. All cell lines were originated from malignant pleural effusions in women with metastatic breast cancer.

$R N A$ isolation and RT-PCR. Total RNA was isolated from the breast cancer cell lines using the Rneasy kit (Qiagen, UK). Total RNA isolated from cells was first treated with DNase to remove any contaminating genomic DNA, ethanol precipitated and reverse-transcribed using Clontech Advantage RT-PCR kit (Clontech). PCR was performed on the cDNA using a sense primer in exon 10 of EMR2 (5'-CGATTCT TCGACAAAGTCCAGGACC-3') and an antisense primer (5'-TGGGTCACCAGATTTCTGTGCCTG-3') in exon 12. As a positive control, a housekeeping gene G6PDH was amplified using the primers (5'-ATGGGGAAGGTGAA GGTCG-3' and 5'-GGGGTCATTGATGGCAACAATA-3') to give a product of $\sim 100 \mathrm{bp}$. As a negative control, total RNA without reverse transcriptase treatment was used to exclude the presence of genomic DNA contamination.
Patients. Between 1990 and 1993, normal tissue derived from breast reductions $(n=10)$ and tissues from 69 cases of DCIS and 272 patients with invasive breast carcinoma undergoing surgery at the John Radcliffe Hospital (Oxford, UK) were collected. Patients ranged in age from 29 to 83 years (median: 57 years) for invasive cancer and from 33 to 75 years (median: 56 years) for DCIS. Cancer patients with distant metastases were excluded from the analysis. Tumours were treated by simple mastectomy $(n=67$ for invasive and 14 for DCIS) or wide local excision $(n=203$ for invasive and 55 for DCIS) with axillary node sampling. Grading of ductal carcinomas was performed by specialist breast pathologists trained at a single institution (John Radcliffe Hospital, Oxford, UK) according to the modified Bloom and Richardson method.

Follow-up was performed every 3 months for the first 18 months and then every 18 months, and clinical parameters, relapse-free survival, and overall survival were recorded from the date of surgery. In patients $<50$ years of age, adjuvant cyclophosphamide, methotrexate, and 5-fluorouracil (CMF) were administered if tumours were node-positive, or ERnegative and/or $>3 \mathrm{~cm}$ in the greatest dimension. Patients $>50$ years with estrogen receptor (ER)-negative, node-positive tumours also received CMF. Tamoxifen was given for 5 years to patients with ER-positive tumours. The median follow-up was 11.6 years (range: 0.02-17.5 years), in which there were 121 relapses and 101 deaths for invasive, 19 relapse and 2 deaths for DCIS. All patients considered in this study gave their written informed consent.

Tissues. A pilot study using whole sections from 10 normal tissue samples, 10 pure DCIS, and 10 pure invasive tumours were examined to determine the pattern and variation of EMR2 expression. To investigate the relationship between the localization and intensity of EMR2 expression with various clinicopathological factors, tissue microarrays from 69 pure in situ carcinomas and 272 invasive carcinomas, as previously described (16) were examined. Sections $(5 \mu \mathrm{m})$ were cut from paraffin-embedded tissues, placed on polylysine-coated slides, and used for immunohistochemical analysis. Intrinsic tumour subsets of luminal, basal, HER2 and null were defined using the criteria of Nielsen et al (17). This study was approved by the Oxfordshire Clinical Research Ethics Committee (CO02.216).

Immunohistochemistry. No antigen retrieval was performed. Sections were first placed in a $60^{\circ} \mathrm{C}$ oven for $20 \mathrm{~min}$ prior to dewaxing in Citroclear twice for $5 \mathrm{~min}$ and rehydration through graded alcohols. Endogenous peroxidase was quenched with the Dako peroxidase block (Dako, UK) applied for $5 \mathrm{~min}$ and then rinsed with Tris buffered saline (TBS) solution. Incubation with $10 \%$ horse serum in TBS for 30 min was then performed to block non-specific binding of the antibody. The primary 2A1 antibody (18) (Clone 2A1, Serotec, UK) that recognises an epitope approximately 200 amino acid residues from the TM7 region of EMR2 (Fig. 1A) was applied to the sections at a concentration of $10 \mu \mathrm{g} / \mathrm{ml}$ in $10 \%$ horse serum and incubated for $90 \mathrm{~min}$ at room temperature (RT) before being rinsed again in TBS solution. The secondary rabbit anti-mouse peroxidase labeled polymer from the Envision HRP kit (Dako), was 


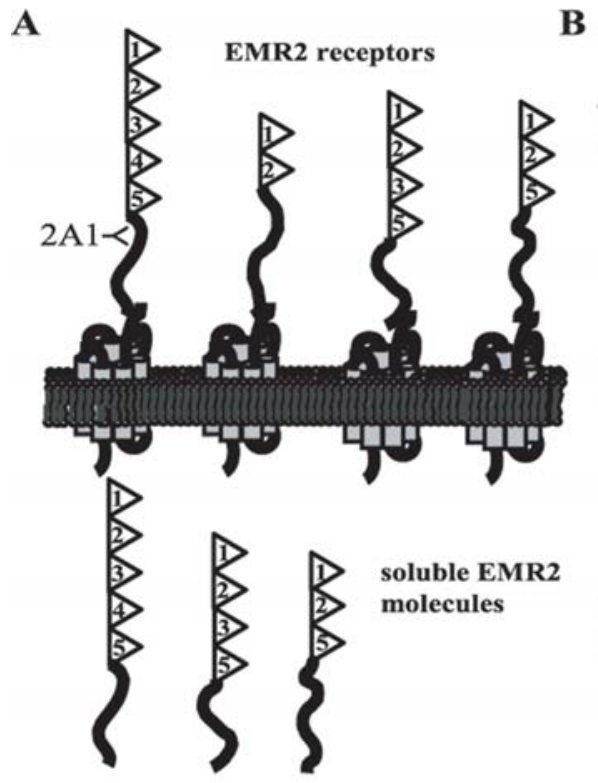

B

C
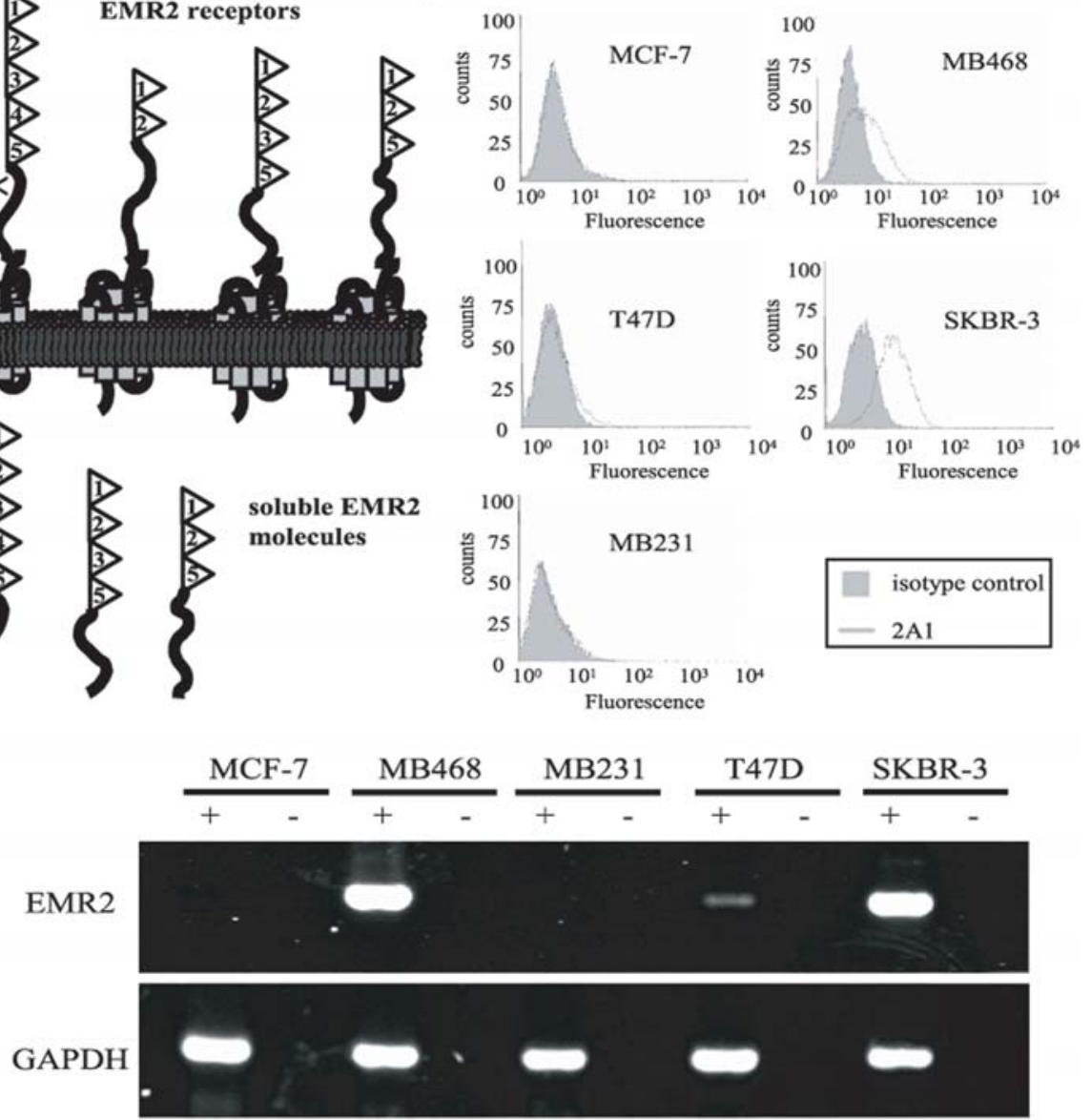

$\mathbf{D}$
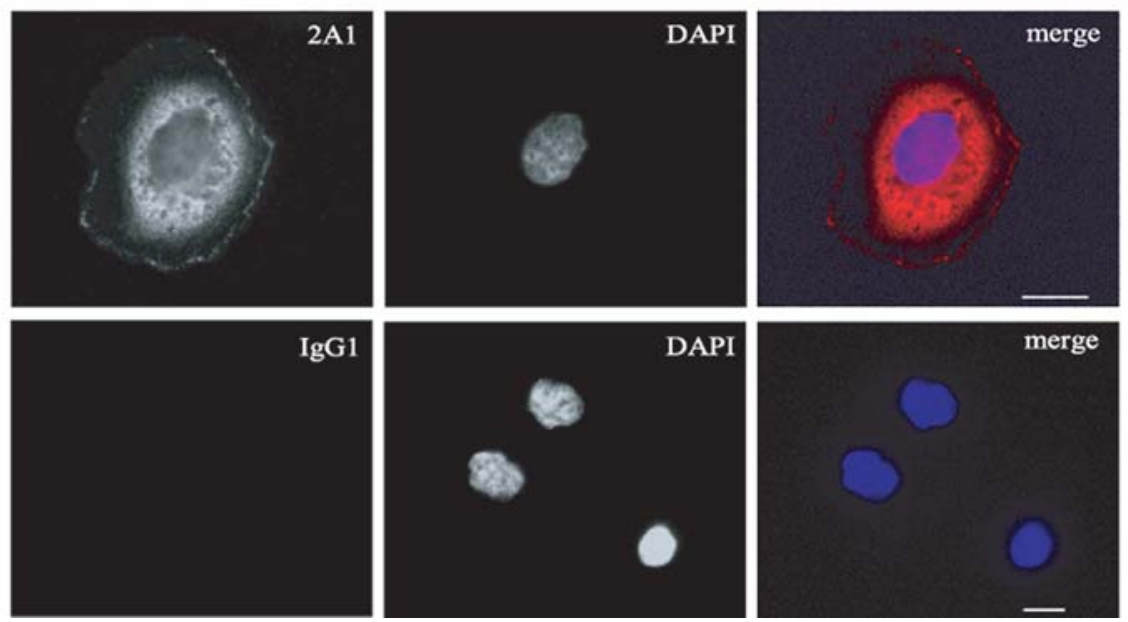

Figure 1. Expression of EMR2 in breast cancer cell lines. (A) Schematic diagram of EMR2 molecules characterized by variable numbers of extracellular EGF-like domains (triangles with numbers), a mucin-like stalk and a 7-transmembrane region. There are several different membrane-anchored and soluble isoforms due to alternative splicing. The monoclonal antibody $2 \mathrm{~A} 1$ recognizes an epitope $\sim 200$ residues from the $7 \mathrm{TM}$ region. (B) Breast cancer cell lines evaluated by FACS analysis showed surface EMR2 expression by mAb 2A1, followed by an anti-mouse PE conjugated secondary Ab. The filled regions correspond to the isotype matched $\mathrm{Ab}$ control. The histogram plots are representative of three separate experiments. (C) The expression levels of EMR2 mRNA in breast cancer cell lines were determined by RT-PCR analysis. + indicates the RT samples; whereas - indicates the non-RT negative control. Data showed the RT-PCR results using EMR2-specific (upper panel) or GAPDH-specific primers (lower panel), which acts as an internal control. (D) Confocal microscopy of SKBR3 cells seeded onto glass coverslips. EMR2 was detected using 2A1 (red, top micrographs). Nuclei were labelled using DAPI (blue). Specificity was confirmed using an IgG1 isotype control (bottom micrographs). The bar represents $5 \mu \mathrm{m}$.

applied for $30 \mathrm{~min}$ at RT. After a further wash, the colour was developed by 5 min incubation with 3,3-diaminobenzidine solution (Dako). Sections were counterstained with haematoxylin and mounted in Aquamount (BDH-Laboratory Supplies, UK).
Immunohistochemical evaluation. Positive controls were infiltrating tumour-associated macrophages (TAMs) and an isotype $\operatorname{IgG} 1$ control antibody (Clone D1.3, in-house hybridoma) was used as a negative control. The tumours were scored by two observers using a conference light microscope 

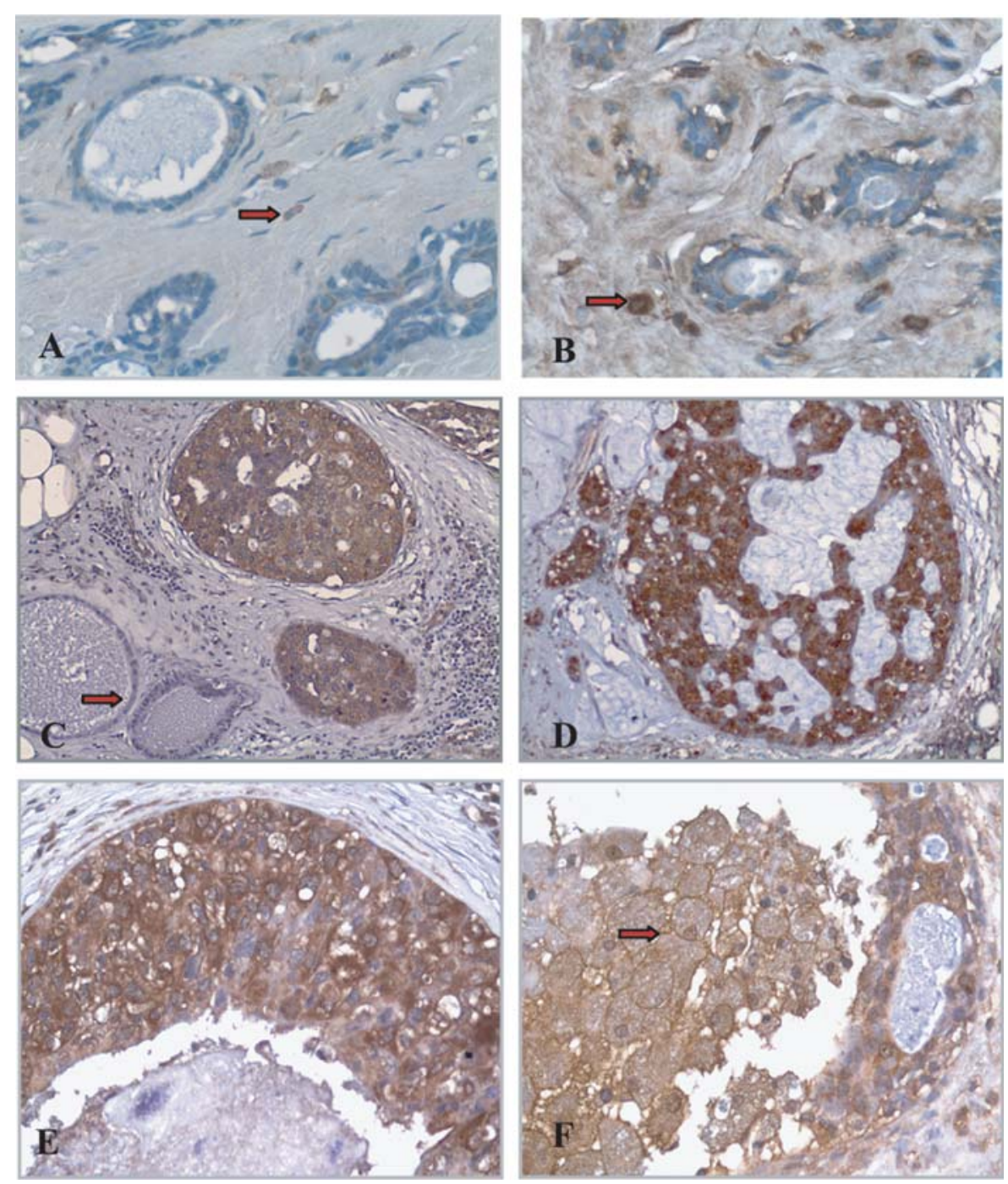

Figure 2. EMR2 expression in normal breast tissue and DCIS. (A and B) Normal breast lobular epithelium shows little EMR2 staining by immunohistochemistry. The surrounding inflammatory cells with the morphology of resident macrophages (arrows) show positive membrane staining. (original magnification, x100). (C) Normal ductal epithelium (arrow) in the vicinity of in situ cancer. The normal ducts appear negative, whereas the in situ neoplastic epithelium expresses EMR2 (original magnification, x100). (D-F) EMR2 expression by ductal carcinoma in situ, also showing additional membrane immunoreactivity of macrophages (arrow) within ducts [original magnification, x100, x400, x400 in (D, E and F) respectively].

utilizing a semi-quantitative system, as previously reported (19) based on cytoplasmic and/or nuclear expression of chromogen intensity: 0, negative staining; 1 , weak staining; 2, moderate staining; and 3, strong staining; with the proportion of cells staining also recorded for percentage as follows: 0 , no cells staining positive for EMR2; $1, \leq 10 \%$ cells staining positive; $2,11-50 \%$ positive cells; $3,51-80 \%$ positive cells; and $4, \geq 80 \%$ positive cells.

Wide-field and confocal immunofluorescence. SKBR3 or MB468 cells were seeded onto glass coverslips. The medium was removed and the cells washed in PBS. The cells were fixed in $4 \%$ paraformaldehyde/PBS on ice for $20 \mathrm{~min}$, quenched with $5 \% \mathrm{BSA} / \mathrm{PBS}$ and then permeabilised and blocked with $0.1 \%$ saponin $/ 5 \%$ goat serum $/ 1 \%$ BSA for $30 \mathrm{~min}$. Paraffin embedded tissue microarrays were placed in a $60^{\circ} \mathrm{C}$ oven for $20 \mathrm{~min}$ prior to dewaxing in Histoclear twice for $5 \mathrm{~min}$ and rehydration through graded alcohols. Primary Ab (2A1) was added at a concentration of $10 \mu \mathrm{g} / \mathrm{ml}$ for a further $90 \mathrm{~min}$.
After washing the cells in PBS, a secondary goat anti-mouseCy3 conjugated $\mathrm{F}\left(\mathrm{ab}^{\prime}\right)_{2} \mathrm{Ab}$ (Jackson) was added $(5 \mu \mathrm{g} / \mathrm{ml}$ in blocking buffer) for a further $30 \mathrm{~min}$. After washing in PBS, the coverslips were lifted and mounted onto glass slides using Vectashield (H-1000) anti-fade mounting medium. For nuclear staining, cells were incubated with DAPI $(1 \mu \mathrm{g} / \mathrm{ml}$ in PBS) for $5 \mathrm{~min}$, before mounting in Vectashield. Widefield fluorescent images were taken on an inverted Zeiss Axiovert 200 fluorescent microscope using a Plan-Apochrome 63X oil immersion lens and the following filters: s360/40, s457/50 for DAPI and s555/28, s617/73 for Cy-3. Analysis by confocal laser scanning microscopy was performed using LaserSharp

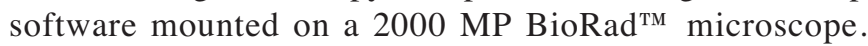
Representative pictures of slides were collected, prior to being imported to Metamorph ${ }^{\mathrm{TM}}$ version 5.0 software for analysis.

Statistical analysis. The Chi-square test was used to test for independence of variables including categorised continuous variables. The log-rank test was used to test for differences in 
Table I. Correlation analyses between intensity of cytoplasmic EMR2 staining and clinicopathological parameters studied by tissue microarray in 69 patients with DCIS.

\begin{tabular}{|c|c|c|c|}
\hline & EMR2 negative & EMR2 positive & $\mathrm{p}$ \\
\hline No. of patients & 42 & 27 & \\
\hline \multicolumn{4}{|l|}{ Age } \\
\hline$<50$ & 12 & 5 & \multirow[t]{2}{*}{0.34} \\
\hline$\geq 50$ & 30 & 22 & \\
\hline \multicolumn{4}{|l|}{ In situ grade } \\
\hline I & 6 & 3 & \multirow[t]{3}{*}{0.32} \\
\hline II & 11 & 12 & \\
\hline III & 25 & 12 & \\
\hline \multicolumn{4}{|l|}{ Estrogen receptor } \\
\hline Negative & 6 & 6 & \multirow[t]{2}{*}{0.64} \\
\hline Positive & 2 & 4 & \\
\hline \multicolumn{4}{|l|}{ Adjuvant hormone } \\
\hline Negative & 27 & 19 & \multirow[t]{2}{*}{0.70} \\
\hline Positive & 14 & 8 & \\
\hline \multicolumn{4}{|l|}{ Surgery } \\
\hline Lumpectomy & 35 & 20 & \multirow[t]{2}{*}{0.35} \\
\hline Mastectomy & 7 & 7 & \\
\hline \multicolumn{4}{|l|}{ Log-rank test } \\
\hline \multicolumn{2}{|c|}{ Relapse-free survival } & & 0.16 \\
\hline Overall survival & & & 0.83 \\
\hline
\end{tabular}

survival and Cox proportional hazard model for multivariate models of survival. All statistics were performed using the Stata package release 10.0 (Stata Corporation, TX, USA).

\section{Results}

EMR2 expression in breast cancer cell lines. A number of breast cancer cell lines were evaluated for the presence of EMR2 by RT-PCR and FACS analysis. FACS analysis showed that two of five cell lines (MB468, and SK-BR3) highly expressed EMR2, while a third cell line, T47D, displayed a weak cell surface EMR2 staining (Fig. 1B). In addition, the expression levels of EMR2 protein identified on FACS staining correlated well with the levels of EMR2specific mRNA, as determined by RT-PCR (Fig. 1C). In order to further confirm the FACS data and to provide an indication of the cellular location of EMR2 in breast cancer cells, fluorescence microscopy was performed on fixed, permeabilised SK-BR3 cells. Fluorescent microscopy demonstrated peripheral membrane and significant perinuclear (most likely endoplasmic reticulum) staining (Fig. 1D) consistent with the location expected for a transmembrane receptor.

Pattern of EMR2 staining in normal breast and in situ and invasive breast carcinomas. Tissue sections of normal breast tissues and tissue microarrays of in situ and invasive breast
Table II. Correlation analyses between intensity of nuclear EMR2 staining and clinicopathological parameters studied by tissue microarray in 69 patients with DCIS.

\begin{tabular}{|c|c|c|c|}
\hline & EMR2 negative & EMR2 positive & $\mathrm{p}$ \\
\hline No. of patients & 48 & 21 & \\
\hline \multicolumn{4}{|l|}{ Age } \\
\hline$<50$ & 13 & 4 & \multirow[t]{2}{*}{0.48} \\
\hline$\geq 50$ & 35 & 17 & \\
\hline \multicolumn{4}{|l|}{ In situ grade } \\
\hline I & 7 & 2 & \multirow[t]{3}{*}{0.71} \\
\hline II & 17 & 6 & \\
\hline III & 24 & 13 & \\
\hline \multicolumn{4}{|l|}{ Estrogen receptor } \\
\hline Negative & 8 & 6 & \multirow[t]{2}{*}{0.70} \\
\hline Positive & 8 & 4 & \\
\hline \multicolumn{4}{|l|}{ Adjuvant hormone } \\
\hline Negative & 33 & 13 & \multirow[t]{2}{*}{0.50} \\
\hline Positive & 14 & 8 & \\
\hline \multicolumn{4}{|l|}{ Surgery } \\
\hline Lumpectomy & 39 & 16 & \multirow[t]{2}{*}{0.63} \\
\hline Mastectomy & 9 & 5 & \\
\hline \multicolumn{4}{|l|}{ Log-rank test } \\
\hline \multicolumn{2}{|c|}{ Relapse-free survival } & & 0.18 \\
\hline Overall survival & & & 0.37 \\
\hline
\end{tabular}

carcinomas were stained by standard immunohistochemistry. Confocal microscopy was also performed on tissue microarray sections to further validate the location of this staining. EMR2 was not seen in normal breast tissue epithelium, but was noted in resident macrophages (Fig. 2A and B). In contrast, EMR2 was strongly expressed in both the cytoplasm and in the nucleus of both the in situ (Fig. 2C-F) and invasive (Fig. 3A-H) neoplastic cells (Tables I and II). The in situ breast cancers expressed EMR2, not only in neoplastic epithelial cells, but also in macrophages present within the ducts (Fig. 2F).

Reassuringly, EMR2 was not expressed in a subset of invasive breast carcinoma (Fig. 3C and D, Tables III and Fig. 4). In most EMR2 positive cases the expression was localised to the cell membrane and cytoplasm (Fig. 3A, B and E). However, in 90 cases (33\%) the nucleus appeared to stain strongly (Fig. 3F and G), showing nucleolar exclusion on confocal microscopy to indicate specificity (Fig. $3 \mathrm{H}$ ). In addition, the associated stromal inflammatory cell infiltrate (Fig. 3B-D) showed strong immunoreactivity for EMR2, indicating that tumour-associated macrophages expressed this antigen in significant quantities. Non-myeloid inflammatory cells, including plasma cells, did not express EMR2 (Fig. 3B inset), consistent with previously published data showing that EMR2 expression is restricted to neutrophils, macrophages and dendritic cells. 

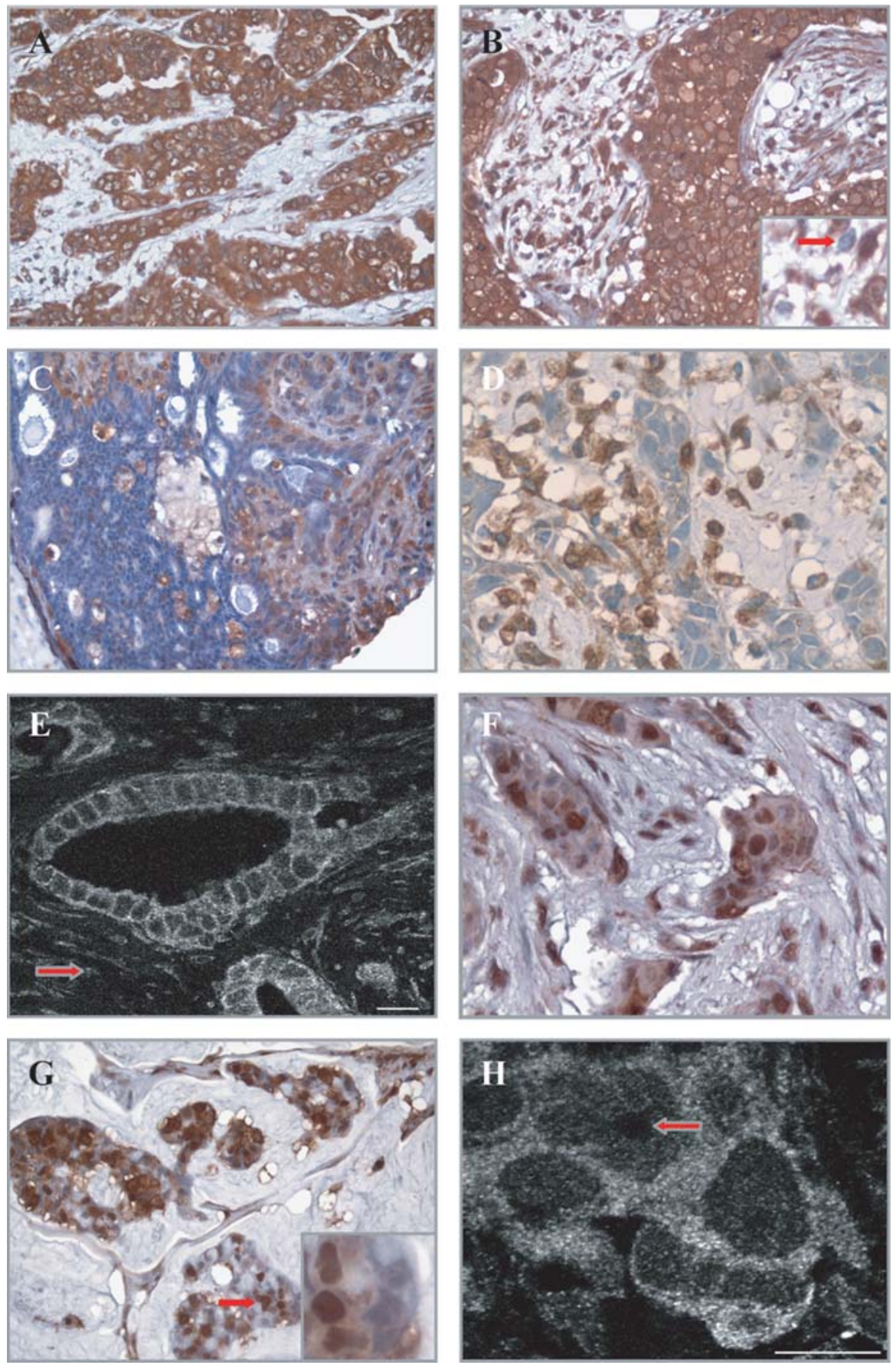

Figure 3. EMR2 expression in invasive breast tissue. (A and B) EMR2 is expressed by infiltrating tumour cells, tumour-associated inflammatory cells and stromal cells. Not all inflammatory cells (B, inset) express EMR2 (original magnification, x200). (C and D) Immunohistochemistry showing that EMR2 is not expressed in all invasive breast cancers. EMR2 is expressed by infiltrating inflammatory cells, which act as an internal control (original magnification, x100, x200). (E) Confocal microscopic analysis of invasive breast cancer. The red arrow indicates expression of EMR2 inflammatory cells in stromal regions. ( $F$ and G) Nuclear staining in invasive breast carcinoma (inset in $G$, original magnification, $x 400$ ). (H) An example of confocal microscopic analysis of invasive breast cancer showing nuclear and cytoplasmic staining. Note exclusion of signal in the nucleolus (arrow). The bar represents $5 \mu \mathrm{m}$.

EMR2 was significantly up-regulated in the cytoplasmic and nuclear compartments of both DCIS $(\mathrm{p}=0.015$ and $\mathrm{p}<0.04$, respectively) and invasive carcinoma $(\mathrm{p}=0.0003$ and $\mathrm{p}<0.0002$, respectively) compared with normal control and significantly higher in invasive compared with in situ diseases $(\mathrm{p}=0.007$ and $\mathrm{p}<0.0001$, respectively). There was no significant difference between expression of EMR2 in the nucleus or cytoplasm between conventional histological types or between intrinsic tumour phenotypes (all $\mathrm{p}>0.05$ ).
Relationship between tumour EMR2 protein expression, clinicopathological parameters and patient survival. There was no significant positive correlation between cytoplasmic EMR2 expression in DCIS and tumour grade $(\mathrm{p}=0.32)$, patient age $(\mathrm{p}=0.34)$, ER expression $(\mathrm{p}=0.64)$, type of surgical treatment $(\mathrm{p}=0.35)$, relapse-free survival $(\mathrm{p}=0.16)$ or overall survival ( $\mathrm{p}=0.83$ ) (Table I). Nuclear EMR2 expression in DCIS showed no significant clinicopathological correlations either (Table II). 
Table III. Correlation analyses between intensity of cytoplasmic EMR2 staining and clinicopathological parameters studied by tissue microarray in 272 patients with invasive breast carcinoma.

\begin{tabular}{|c|c|c|c|}
\hline & EMR2 negative & EMR2 positive & $\mathrm{p}$ \\
\hline No. of patients & 116 & 156 & \\
\hline \multicolumn{4}{|l|}{ Age } \\
\hline$<50$ & 33 & 45 & \multirow[t]{2}{*}{0.95} \\
\hline$\geq 50$ & 82 & 110 & \\
\hline \multicolumn{4}{|l|}{ Nodal status } \\
\hline Negative & 60 & 93 & \multirow[t]{2}{*}{0.20} \\
\hline Positive & 55 & 62 & \\
\hline \multicolumn{4}{|l|}{ Tumor size } \\
\hline$\leq 2 \mathrm{~cm}$ & 67 & 80 & \multirow[t]{2}{*}{0.28} \\
\hline$>2 \mathrm{~cm}$ & 48 & 75 & \\
\hline \multicolumn{4}{|l|}{ Grade } \\
\hline I & 21 & 14 & \multirow{3}{*}{$\begin{array}{l}0.06^{\mathrm{a}} \\
(+\mathrm{ve})\end{array}$} \\
\hline II & 60 & 98 & \\
\hline III & 29 & 44 & \\
\hline \multicolumn{4}{|c|}{ Estrogen receptor } \\
\hline Negative & 35 & 54 & \multirow[t]{2}{*}{0.45} \\
\hline Positive & 80 & 101 & \\
\hline \multicolumn{4}{|l|}{ EGFR } \\
\hline Negative & 46 & 60 & \multirow[t]{2}{*}{0.84} \\
\hline Positive & 67 & 92 & \\
\hline \multicolumn{4}{|c|}{ Test for survival } \\
\hline \multicolumn{2}{|c|}{ Relapse-free survival } & & 0.73 \\
\hline Overall surv & & & 0.57 \\
\hline
\end{tabular}

Cytoplasmic EMR2 expression in invasive breast carcinomas was associated with high tumour grade $(p=0.06)$, but not with patient age $(\mathrm{p}=0.95)$, nodal status $(\mathrm{p}=0.20)$, tumour size $(\mathrm{p}=0.28)$, ER status $(\mathrm{p}=0.45)$, relapse-free $(\mathrm{p}=0.73)$ or overall survival ( $\mathrm{p}=0.57$ ) (Table III and Fig. 4A). Nuclear EMR2 expression was significantly inversely correlated with tumour size $(p=0.03)$, but not with patient age $(p=0.63)$, nodal status $(\mathrm{p}=0.21)$, tumour grade $(\mathrm{p}=0.12)$ or ER status $(\mathrm{p}=0.15)$ (Table IV). Nuclear EMR2 staining was positively associated with longer relapse-free $(\mathrm{p}=0.08)$ and overall survival ( $\mathrm{p}=0.01$ ) (Fig. 4B). A multivariate statistic analysis of nuclear and cytoplasmic EMR2 expression did not confered any independent prognostic information, either for relapse-free or overall survival (data not shown).

\section{Discussion}

A role for leukocyte GPCRs in the development and progression of cancers has been well established (20). For

\section{$\mathbf{A}$}

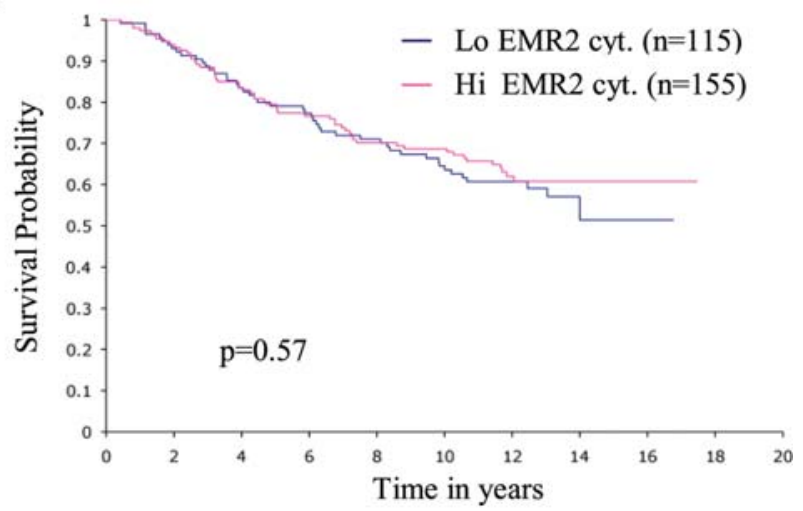

B

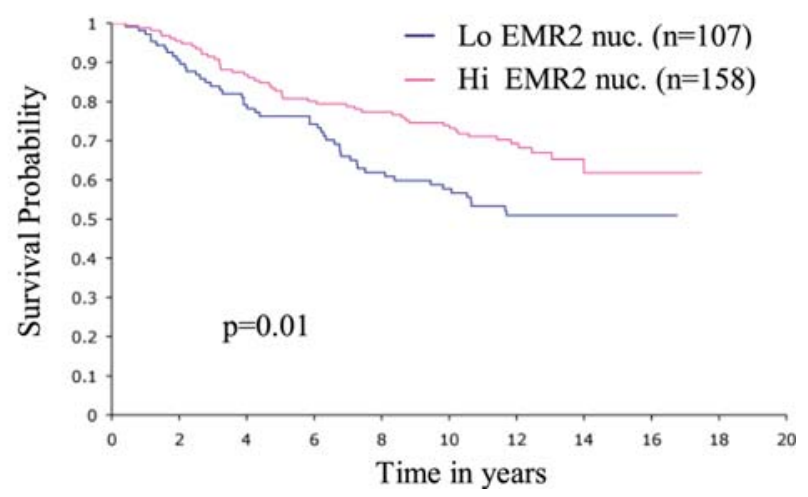

Figure 4. The relationship of EMR2 expression and patient survival. Kaplan-Meier survival curves of overall survival in patients stratified by (A) cytoplasmic (cyt.) and (B) nuclear (nuc.) EMR2 expression. Lo, low; Hi, high.

example, the chemokine receptors CXCR4 and CCR7 can induce chemotactic and invasive responses in breast cancer cells by mediating actin polymerisation and pseudopodia formation (21). This receptor modulation may be at least partially responsible for organ-specific metastasis in cancer progression, where cancer cells expressing chemokine receptors are able to respond to chemokine gradients in target organs. However, the chemokine receptors are not likely to be the only cell surface molecules involved in modulating chemotaxis, invasiveness or metastasis in malignancy. In fact, in recent years another large family of 7TM receptors, the adhesion GPCRs have been shown to modulate invasive characteristics in human cancer (12). It is the identification of new molecules, such as these, which may improve the understanding of the aetiology, pathogenesis, treatment and prevention of human cancer.

In this study, we report the novel observation that EMR2, a normally leukocyte-restricted adhesion GPCR, is present in certain breast cancer cell lines as well as in normal and neoplastic breast tissue. We demonstrate an increase in EMR2 expression during the transition from normal, to in situ and invasive carcinomas and note that cytoplasmic expression correlates with pathological grade in invasive carcinoma, suggesting that EMR2 may play an important biological role in the evolution of breast carcinogenesis. Data in our 
Table IV. Correlation analyses between intensity of nuclear EMR2 staining and clinicopathological parameters studied by tissue microarray in 268 patients with invasive breast carcinoma.

\begin{tabular}{|c|c|c|c|}
\hline & EMR2 negative & EMR2 positive & $\mathrm{p}$ \\
\hline No. of patients & 109 & 159 & \\
\hline \multicolumn{4}{|l|}{ Age } \\
\hline$<50$ & 32 & 43 & \multirow[t]{2}{*}{0.63} \\
\hline$\geq 50$ & 75 & 115 & \\
\hline \multicolumn{4}{|l|}{ Nodal status } \\
\hline Negative & 56 & 95 & \multirow[t]{2}{*}{0.21} \\
\hline Positive & 51 & 63 & \\
\hline \multicolumn{4}{|l|}{ Tumor size } \\
\hline$\leq 2 \mathrm{~cm}$ & 49 & 94 & \multirow{2}{*}{$\begin{array}{l}0.03 \\
(+\mathrm{ve})\end{array}$} \\
\hline$>2 \mathrm{~cm}$ & 58 & 64 & \\
\hline \multicolumn{4}{|l|}{ Grade } \\
\hline I & 8 & 26 & \multirow[t]{3}{*}{0.12} \\
\hline II & 65 & 89 & \\
\hline III & 29 & 41 & \\
\hline \multicolumn{4}{|c|}{ Estrogen receptor } \\
\hline Negative & 41 & 7 & \multirow[t]{2}{*}{0.15} \\
\hline Positive & 66 & 111 & \\
\hline \multicolumn{4}{|l|}{ EGFR } \\
\hline Negative & 44 & 61 & \multirow[t]{2}{*}{0.53} \\
\hline Positive & 59 & 96 & \\
\hline \multicolumn{4}{|c|}{ Test for survival } \\
\hline \multicolumn{2}{|c|}{ Relapse-free survival } & & $0.08^{\mathrm{a}}$ \\
\hline Overall surv & & & $0.01^{\mathrm{a}}$ \\
\hline
\end{tabular}

aDenotes significantly longer survival.

laboratory indicate that cross-linking EMR2 with monoclonal antibody on the cell surface of leukocytes, or overexpressing EMR2 in cell lines, results in cellular activation and an enhanced migration index in response to certain chemotactic ligands (4). One of the ligands for EMR2 is chondroitin sulphate (15), a glycosaminoglycan present in large quantities in the peritumoural stroma of breast (22). Thus, we hypothesize that breast cancer cells of high grade are able to acquire membrane or cytoplasmic EMR2 and undergo metabolic 'activation' by binding to the high levels of ligand in the surrounding tissue, resulting in cellular migration. Careful examination of the pattern of immunohistological staining of EMR2 and its corresponding chondroitin sulphate ligand in whole frozen section tissue sections (15) needs to be evaluated in this regard.

In addition to the cytoplasmic expression of EMR2, the 'aberrant' expression of EMR2 in the nucleus was found to be a protective variable in relation to overall patient survival, suggesting that EMR2 might play a role in breast epithelial malignancy. Nuclear expression of many GPCRs is a well known, although poorly understood phenomenon, which has been proposed to be essential for many physiological cellular processes including gene transcription and cell proliferation (23). In this respect, intracellular signaling is thought to occur following endocytosis and nuclear translocation of peripherally ligated GPCRs; or following the activation of nuclear located GPCRs by endogenously produced non-secreted ligands (24). Nuclear localization has been documented in many GPCRs including the PTH receptor (25), a class B GPCR with 7TM sequence similarities to EMR2.

In addition, nuclear localization of CXCR4 (an unrelated GPCR) has been reported in a number of human cancers including hepatocellular carcinoma (26), non-small cell carcinoma of the lung (27), colorectal carcinoma (28) and nasopharyngeal carcinoma (29). Thus, EMR2 is a new example of GPCR expressed in the nucleus of cancer cells. The underlying mechanism resulting in targeting of a membrane receptor to the nucleus is a subject of debate, but may be attributed to a nuclear localization sequence (NLS), which has been clearly identified in some GPCRs in the eighth helix or third intracellular loops (30). Although EMR2 has no such identifiable sequence, other unidentified sequence motifs might still promote nuclear importation of proteins (31). A possible additional mechanism involves association of the receptor with a carrier protein containing an NLS, such as is the case with the PTH receptor where the NLS might be provided by its ligand (PTHrP) (23). Clearly, this phenomenon demonstrated by EMR2 requires further in depth investigation.

Finally, we noted that membrane localized EMR2 was also expressed in high quantites by tumour-associated macrophages, inflammatory cells known to play a crucial part in neoplasia, by producing substances that promote tumour growth, cell survival, angiogenesis and invasiveness (32). This raises the possibility that EMR2, such as soluble CD97 (6), might have a similar function in breast cancer, whereby tumour cells and/or tumour-associated macrophages cleave EMR2 from their cell membranes into the extracellular milleau, thereby modulating new vessel formation and therefore ultimate tumour behaviour.

We identified EMR2, a leukocyte-restricted adhesionGPCR, in a number of breast cancer cell lines and in normal and neoplastic breast tissue. In addition, we show that there is strong expression of EMR2 in inflammatory cells as part of the surrounding stromal reaction. Strong cytoplasmic EMR2 expression appeared more prevalent in higher grade invasive and non-invasive tumours. Of note, strong nuclear staining of EMR2 showed an inverse pattern in invasive disease, being more prevalent in lower grade tumours, where it appeared to be a biomarker of improved patient survival.

\section{Acknowledgements}

This study was supported by the Nuffield Foundation to J.Q.D. and research grants from the Chang Gung Memorial Hospital (CMRPD160063 and CMRPD160383) to H.-H.L.

\section{References}

1. Yona S, Lin HH, Siu WO, Gordon S and Stacey M: AdhesionGPCRs: emerging roles for novel receptors. Trends Biochem Sci 33: 491-500, 2008. 
2. Leemans JC, te Velde AA, Florquin S, et al: The epidermal growth factor-seven transmembrane (EGF-TM7) receptor CD97 is required for neutrophil migration and host defense. J Immunol 172: 1125-1131, 2004.

3. Lin HH, Faunce DE, Stacey M, et al: The macrophage F4/80 receptor is required for the induction of antigen-specific efferent regulatory $\mathrm{T}$ cells in peripheral tolerance. J Exp Med 201: 1615-1625, 2005.

4. Yona S, Lin HH, Dri P, et al: Ligation of the adhesion-GPCR EMR2 regulates human neutrophil function. FASEB J 22: 741-751, 2008.

5. Capasso M, Durrant LG, Stacey M, Gordon S, Ramage J and Spendlove I: Costimulation via CD55 on human $\mathrm{CD}^{+} \mathrm{T}$ cells mediated by CD97. J Immunol 177: 1070-1077, 2006.

6. Wang T, Ward Y, Tian L, et al: CD97, an adhesion receptor on inflammatory cells, stimulates angiogenesis through binding integrin counterreceptors on endothelial cells. Blood 105: 2836-2844, 2005.

7. Jaspars LH, Vos W, Aust G, Van Lier RA and Hamann J: Tissue distribution of the human CD97 EGF-TM7 receptor. Tissue Antigens 57: 325-331, 2001.

8. Aust G, Eichler W, Laue S, et al: CD97: a dedifferentiation marker in human thyroid carcinomas. Cancer Res 57: 1798-1806, 1997.

9. Aust G, Steinert M, Schutz A, et al: CD97, but not its closely related EGF-TM7 family member EMR2, is expressed on gastric, pancreatic, and esophageal carcinomas. Am J Clin Pathol 118: 699-707, 2002.

10. Mustafa T, Klonisch T, Hombach-Klonisch S, et al: Expression of CD97 and CD55 in human medullary thyroid carcinomas. Int J Oncol 24: 285-294, 2004.

11. Galle J, Sittig D, Hanisch I, et al: Individual cell-based models of tumor-environment interactions: Multiple effects of CD97 on tumor invasion. Am J Pathol 169: 1802-1811, 2006.

12. Steinert M, Wobus M, Boltze C, et al: Expression and regulation of CD97 in colorectal carcinoma cell lines and tumor tissues. Am J Pathol 161: 1657-1667, 2002.

13. Lin HH, Stacey M, Hamann J, Gordon S and McKnight AJ: Human EMR2, a novel EGF-TM7 molecule on chromosome $19 \mathrm{p} 13.1$, is closely related to CD97. Genomics 67: 188-200, 2000.

14. Kwakkenbos MJ, Pouwels W, Matmati M, et al: Expression of the largest CD97 and EMR2 isoforms on leukocytes facilitates a specific interaction with chondroitin sulfate on B cells. J Leukoc Biol 77: 112-119, 2005.

15. Stacey M, Chang GW, Davies JQ, et al: The epidermal growth factor-like domains of the human EMR2 receptor mediate cell attachment through chondroitin sulfate glycosaminoglycans. Blood 102: 2916-2924, 2003.

16. Campo L, Turley H, Han C, et al: Angiogenin is up-regulated in the nucleus and cytoplasm in human primary breast carcinoma and is associated with markers of hypoxia but not survival. J Pathol 205: 585-591, 2005.
17. Nielsen TO, Hsu FD, Jensen K, et al: Immunohistochemical and clinical characterization of the basal-like subtype of invasive breast carcinoma. Clin Cancer Res 10: 5367-5374, 2004.

18. Chang GW, Stacey M, Kwakkenbos MJ, Hamann J, Gordon S and Lin HH: Proteolytic cleavage of the EMR2 receptor requires both the extracellular stalk and the GPS motif. FEBS Lett 547: 145-150, 2003.

19. Tan EY, Campo L, Han C, et al: BNIP3 as a progression marker in primary human breast cancer; opposing functions in in situ versus invasive cancer. Clin Cancer Res 13: 467-474, 2007.

20. Dhanasekaran N, Heasley LE and Johnson GL: G protein-coupled receptor systems involved in cell growth and oncogenesis. Endocr Rev 16: 259-270, 1995.

21. Muller A, Homey B, Soto H, et al: Involvement of chemokine receptors in breast cancer metastasis. Nature 410: 50-56, 2001.

22. Suwiwat S, Ricciardelli C, Tammi R, et al: Expression of extracellular matrix components versican, chondroitin sulfate, tenascin, and hyaluronan, and their association with disease outcome in node-negative breast cancer. Clin Cancer Res 10: 2491-2498, 2004.

23. Gobeil F, Fortier A, Zhu T, et al: G-protein-coupled receptors signalling at the cell nucleus: an emerging paradigm. Can J Physiol Pharmacol 84: 287-297, 2006.

24. Re R: The nature of intracrine peptide hormone action. Hypertension 34: 534-538, 1999.

25. Watson PH, Fraher LJ, Natale BV, Kisiel M, Hendy GN and Hodsman AB: Nuclear localization of the type 1 parathyroid hormone/parathyroid hormone-related peptide receptor in MC3T3-E1 cells: association with serum-induced cell proliferation. Bone 26: 221-225, 2000.

26. Shibuta K, Mori M, Shimoda K, Inoue H, Mitra P and Barnard GF: Regional expression of CXCL12/CXCR4 in liver and hepatocellular carcinoma and cell-cycle variation during in vitro differentiation. Jpn J Cancer Res 93: 789-797, 2002.

27. Spano JP, Andre F, Morat L, et al: Chemokine receptor CXCR4 and early-stage non-small cell lung cancer: pattern of expression and correlation with outcome. Ann Oncol 15: 613-617, 2004.

28. Nielsen CK, Campbell JI, Ohd JF, et al: A novel localization of the G-protein-coupled CysLT1 receptor in the nucleus of colorectal adenocarcinoma cells. Cancer Res 65: 732-742, 2005.

29. Wang N, Wu QL, Fang Y, et al: Expression of chemokine receptor CXCR4 in nasopharyngeal carcinoma: pattern of expression and correlation with clinical outcome. J Transl Med 3: 26,2005 .

30. Lee DK, Lanca AJ, Cheng R, et al: Agonist-independent nuclear localization of the Apelin, angiotensin AT1, and bradykinin B2 receptors. J Biol Chem 279: 7901-7908, 2004.

31. Christophe D, Christophe-Hobertus C and Pichon B: Nuclear targeting of proteins: how many different signals? Cell Signal 12: 337-341, 2000.

32. van der Bij GJ, Oosterling SJ, Meijer S, Beelen RH and van Egmond M: The role of macrophages in tumor development. Cell Oncol 27: 203-213, 2005. 